\section{Detecting nuclear particles}

Nuclear Electronics. By P. W. Nicholson. Pp. xiv +388. (Wiley: London and New York, April 1974.) £8.75.

THIS book is aimed specifically at physics graduates needing to use nuclear detectors. The first two chapters are introductory accounts of the principles involved in detecting nuclear particles and of the basic ideas of pulse circuits; these chapters are provided on the assumption, which unfortunately is probably correct, that most undergraduate courses contain very little on these topics. The next three chapters give an account of the general problem of the amplification of nuclear pulses and the methods of minimising the effect of noise. This is the most attractive part of the book as the author gives particularly clear explanations of the problems which arise and the methods used to solve them. The remaining chapters deal with discrminators, digitising techniques, for both amplitudes and time differences, and various more specialised circuits. In these sections it is inevitably more dificult to provide any connected chain of argument but the treatment is still reasonably uniform: any theory is given first, the various difficulties and their solution are clearly explained and a well chosen selection of recently published circuits is given as illustration.

This is essentially a text book rather than a practical guide. Brief accounts are included of the operation of some semiconductor devices, but the whole subject of component characteristics and limitations is omitted and there is hardly any mention of practical 'engineering' problems such as interference, effects of stray capacitances or long-term stability.

I think the author has been very successful in carrying out his purpose but unfortunately a detailed theoretical understanding is not sufficient for the experimental physicist, whose problems in practice are of two sorts. First, given a large budget, he has to ask whether commercially available equipment will have the necessary performance; this book will certainly help him to understand manufacturers' specifications but considerable skill and experience in electronics is necessary to solve' those problems for which equipment is not available. Second, and especially for those with inadequate budgets, it is necessary to know how to build circuits with a performance which, though less than 'state of the art', may still be adequate for a particular purpose. So it seems a pity that $\operatorname{Dr}$ Nicholson, by concentrating on the best published circuits, gives little indication of what may be achieved more simply. For example, it would have been worth stressing how easy it is, even for the non- specialist, to use digital integrated circuits in constructing counting equipment.

Despite such omissions, this book will be very useful to anybody working with nuclear counting systems. I noted only a few errors or misprints and the author is to be particularly commended on the clarity of his explanations and of the diagrams.

J. C. BARTON

\section{Counting heads}

A Workbook in Demography. By Roland Pressat. Translated by E. Grebenik with C. A. M. Sym. Pp. 292. (Methuen: London, May 1974.) £7.90.

THE age of the population explosion and its implications for society and for the world's resource base has attracted students in several disciplines to examine demographic problems. This interest is catered for by a growing general literature and by regional and case studies, but the techniques and methodology of demography have been generally somewhat neglected. Many postgraduate students find a need to provide their own basic training and regret the absence of straightforward, general textbooks that explain not only simply concepts but also the practical mechanism of calculating data from raw statistics, illustrating where relevant the major pitfalls for the unwary. Few are as well qualified to undertake this task as Professor Pressat, a mathematician by training, who has done much to define the aims and methods of the quantitative approach to population study. He has provided us with just such a textbook as described above, setting up problems, supplying suitable data and offering means of solution and explanation of significance.

The "exercises"-for this is truly a workbook-have been selected to present real life situations. The examples are spread widely over the confusing variety of situations that confront the beginner and the author brings to them an understandable order and relevance. For the more advanced student, he has sought to provide examples close to the situations faced by research workers. Not all the exercises are mathematical: several depend on descriptive interpretation. Nevertheless, using his early training, he makes the mathematics intelligible to those with only modest numeracy. Although designed for students in the University of Paris and consequently using substantial amounts of French data, the author has nevertheless selected from a wide range of countries, both "advanced" and "developing", and he has included some most interesting Soviet material.

The topics are grouped conceptually, starting with the concept of a population. The reader is then taken through the measurement and comparison of mortality and some of the main problems of such difficulties as the misstatement of age. The exercises include not only contemporary situations but also the techniques of calculating data for past conditions. Anomalies, wherever they occur, are explained at length and in his answers the author makes some interesting comments on contemporary problems. For example, the stress of modern urban living may kill the weaker older people who survived infancy through the advances of modern medicine, whereas in earlier times they would have been winnowed by natural selection in childhood. Nuptiality, disunion (separation?) and re-marriage are dealt with in a similar manner and are followed by examination of fertility, where material is drawn from North American and African experience and used in comparative studies. The final section covers ageing population, infant mortality, birth order and replacement. It is a pity there is nothing on migration.

Treatment is thorough and wherever necessary basic techniques are explained. The student will find the many valuable formulae most useful and the author explains how to use fragmentary and unsatisfactory data by skilful manipulation. This is indispensible as a reference book for anybody interested in population.

\section{R. E. H. MELLOR}

\section{Not so fast}

Chemical Kinetics: Homogeneous Reactions. By N. M. Emanuel' and D. G. Knorre. Translated from Russian by R. Kondor. Translation edited by $D$. Slutzkin. Pp. xii +447 . (Wiley: New York and Toronto; Israel Program for Scientific Translations: Jerusalem, February 1974.) $£ 8$.

THIS is a soundly written book with a broad coverage of topics. It is, however, mainly concerned with work up to and including the early transition state era. As a succinct example, the work of Michael Polanyi on energy surfaces is described, but not that of his son John Polanyi. All this gives the book an oddly out-of-date flavour. Short references to flash photolysis, electron paramagnetic resonance and so on indicate the updating efforts of the second Russian edition (Moscow, 1969) but the general effect still remains. Honours students may look at the book for background material, chemists generally for the mathematical treatments of the concentration-time curves of various complex reactions. But there is nothing here comparable to the general monographs of, say, Kassel or 